\title{
Benchmarking a MOS-based Algorithm on the BBOB-2010 Noiseless Function Testbed
}

\author{
Antonio LaTorre \\ Department of Computer \\ Systems Architecture and \\ Technology \\ Facultad de Informática \\ Universidad Politécnica de \\ Madrid, Spain \\ atorre@fi.upm.es
}

\author{
Santiago Muelas \\ Department of Computer \\ Systems Architecture and \\ Technology \\ Facultad de Informática \\ Universidad Politécnica de \\ Madrid, Spain \\ smuelas@fi.upm.es
}

\author{
José M. Peña \\ Department of Computer \\ Systems Architecture and \\ Technology \\ Facultad de Informática \\ Universidad Politécnica de \\ Madrid, Spain \\ jmpena@fi.upm.es
}

\begin{abstract}
In this contribution, a hybrid algorithm combining Differential Evolution and IPOP-CMA-ES is presented and benchmarked on the BBOB 2010 noiseless testbed. The hybrid algorithm has been constructed within the Multiple Offspring Sampling framework, which allows the seamless combination of multiple metaheuristics in a dynamic algorithm capable of adjusting the participation of each of the composing algorithms according to their current performance. The experimental results show a robust behavior of the algorithm and a good scalability as the dimensionality increases.
\end{abstract}

\section{Categories and Subject Descriptors}

G.1.6 [Numerical Analysis]: Optimization-global optimization, unconstrained optinization; F.2.1 [Analysis of Algorithms and Problem Complexity]: Numerical Algorithms and Problems

\section{General Terms}

Algorithms

\section{Keywords}

Benchmarking of algorithms, Black-box optimization, Continuous optimization, IPOP-CMA-ES, Differential Evolution, Multiple Offspring Sampling

\section{INTRODUCTION}

In this contribution, a hybrid algorithm constructed by means of the Multiple Offspring Sampling (MOS) framework has been applied to the Black Box Optimization 2010 Noiseless Function Testbed. This framework allows the combination of different evolutionary models following an $\mathrm{HRH}$ (High-level Relay Hybrid) approach (according to Talbj's taxonomy, briefly reviewed in Section 2) in which the number of evaluations that each algorithm can carry out is dynamically adjusted. For this paper, the IPOP-CMAE-ES [1] and the Differential Evolution (DE) algorithm [8] have been combined within this framework in a multistart strategy and has been benchmarked on 24 different functions. Detailed results regarding the number of evaluations needed to reach a target function on each dimension along with the CPU times are also given.

\section{ALGORITHM PRESENTATION}

Multiple Offspring Sampling (MOS) is a framework for the development of Dynamic. Hybrid Evolutionary Algorithms [6]. MOS provides the functional formalization necessary to design this type of algorithms, as well as the tools to identify and select the best performing configuration for the problem under study. In this context, the hybridization of several algorithms can lead to the following two situations:

- A collaborative synergy emerges among the different algorithms that improves the performance of the best one when it is used individually.

- A competitive selection of the best one takes place, in which a similar performance (often the same) is obtained with a minimum overhear.

In MOS, a key term is the concept of technique, which is a mechanism, decoupled from the main algorithm, to generate new candidate solutions. This means that, within a MOSbased algorithm, several reproductive mechanisms can be used simultaneously, and it is the main algorithm which selects among the available optimization techniques the most appropriate for the particular problem and search phase. A more concrete definition for these reproductive mechanisms follows:

Defnition 1. A MOS reproductive technique is a mechanism to create new individuals in which: (a) a particular evolutionary algorithm model, (b) an appropriate solution encoding, (c) specific operators (if required), and (d) necessary parameters have been defined.

Furthermore, the use of multiple reproductive mechanisms simultaneously has to be controlled in some way. The MOS framework offers two groups of functions to deal with this 
issue: Quality and Participation functions. The first group of functions evaluate how good a set of new individuals is from the point of view of a desirable characteristic. The second group of functions consider the quality values computed by the first group and adjust the number of new individuals that each reproductive technique will be allowed to generate in the next step of the search. This way, the algorithm is able to dynamically adjust the participation of each of the avajlable techniques and exploit the benefits of each of them at different stages of the search process.

Finally, the Multiple Offspring Sampling framework allows the development of both HTH (High-level Teamwork Hybrid) and HRH (High-level Relay Hybrid) algorithms (according to Talbi's nomenclature [9]). In the case of the HTH algorithms, two metaheuristics are executed in parallel, working at the same time on the resolution of the problem. On the other hand, in the case of the HRH algorithms, two metaheuristics are executed in sequence, one after the other, and changes of the executing algorithm are carried out according to a given policy. As the proposed algorithm is of the HRH type, more attention will be pajd to this type of algorithms. In terms of the MOS framework, the available techniques in a MOS-based HRH hybrid algorithm are used in sequence, one after the other, each of them reusing the output population of the previous technique. This approach fits better when there are non-population-based techniques, such as local searches, as techniques are not constrained to produce a \% of the common population. If different population sizes are used by different techniques, it is the responsibility of the technique to make grow/shrink the population in order to adjust it to its needs and to return a population of an appropriate size to the next technique. For example, if a population-based algorithm is combined with a local search, the latter could select one or more individuals from the output population of the population-based algorithm, modify them as needed and then include them in the original population by means of a predefined elitism procedure. In this type of algorithms, the search process is divided into a fixed number of steps that is established at the beginning of the execution. Each step is assigned an amount of Fitness Evaluations ( $F E s_{i}$ in Algorithm 1), which are distributed by the Participation Function (PF). Each technique can manage its number of allocated FEs at each step of the algorithm $\left(F E \varepsilon_{i}^{(j)}\right)$ in its own particular way. For example, a population-based technique, such as Differential Evolution, could execute several iterations of the algorithm, whereas a Local Search could decide to spend all its assigned evaluations in improving just one individual. The quality of the new individuals of each technique will be averaged at the end of the whole set of evaluations, as the division of the search into generations depends on each of the techniques. A pseudocode of this approach is given in Algorithm 1. Further information about the MOS framework can be found in $[6]$.

In this contribution, an HRH Dynamic algorithm is proposed. This algorithm combines the explorative/exploitative strength of two heuristic search methods, that separately have proven to obtain very competitive results in either low or high dimensional problems. These algorithms are: the IPOP-CMA-ES algorithm [1], the best algorithm of the "Special Session on Real-Parameter Optimization" held at the CEC 2005 Congress, and the DE algorithm [8] which has demonstrated to obtain competitive results when executed

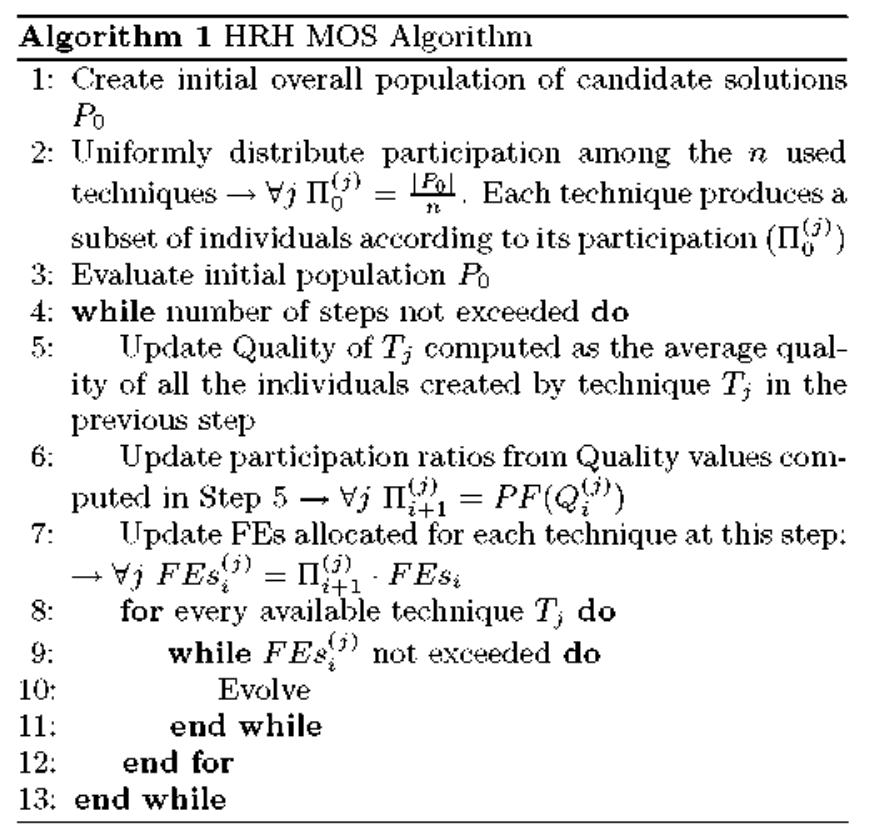

independently and when combined with other algorithms $[3$, 7].

For the adjustment of the participation of each technique in the overall search process, a new Quality Function (QF) has been proposed. This $\mathrm{QF}$ takes into account two desirable characteristics in a search algorithm: the Average Fitness Increment of the newly created individuals after a set of allocated Fitness Evaluations and the number of times that these improvements take place (Equation 1).

$$
\begin{aligned}
& \quad Q_{i}^{(j)}=\left\{\begin{array}{cc}
\Sigma_{i-1}^{(j)} & \text { if } \Sigma_{i-1}^{(j)}>\Sigma_{i-1}^{(k)} \wedge \\
\Gamma_{i-1}^{(j)}>\Gamma_{i-1}^{(k)}
\end{array} \quad \forall j, k \in[1, n]\right. \\
& \Gamma_{i-1}^{(j)} \quad \text { othervise }
\end{aligned} \quad \begin{aligned}
& Q_{i}^{(j)} \equiv \text { Quality of technique } T_{j} \text { in step } i \\
& \Sigma_{i}^{(j)} \equiv \text { Average Fitness Increment of } T_{j} \text { in step } i \\
& \Gamma_{i}^{(j)} \equiv \text { Number of Fitness improvements of } T_{j} \text { in step }:
\end{aligned}
$$

This Quality Function uses the Average Fitness Increment as the effective QF only if there is consensus among both measures. If this is not the case, the raw number of fitness improvements is used. The logic behind this function is that, in some functions, the use of the Average Fitness Increment $\mathrm{QF}$ could be very elitist. In some particular situations, a technique which is not carrying out an effective search could introduce, for some reason, a large increment in the average fitness value of the new individuals. This could be due, for example, to a recombination of poor solutions. In such a case, it is easy for a technique to improve previous solutions. However, it could be more adequate to carry out small changes to good individuals in order to find the right "path" to the global optimum rather than carrying out substantial modifications to poor solutions. For this reason, a consensus of both measures is required in order to apply the more elitist Average Fitness Increment QF. If this is not the case, the number of fitness improvements is used to guarantee a softer adjustment of participation. 
The quality values computed by this QF are used by a Dynamic Participation Function to adjust the number of Fitness Evaluations allocated for each technique at each step (Equation 2). This PF computes, at each step, a trade-off factor for each technique, $\Delta_{i}^{(j)}$, that represents the decrease in participation for the $j-t h$ technique at the $i-t h$ step, for every technique except the best performing ones. These techniques will increase their participation by the sum of all those $\Delta_{i}^{(j)}$ divided by the number of techniques with the best quality values.

$$
\begin{gathered}
P F_{d y n}\left(Q_{i}^{(j)}\right)= \begin{cases}\Pi_{i-1}^{(j)}+\eta & \text { if } j \in b e s t, \\
\Pi_{i-1}^{(j)}-\Delta_{i}^{(j)} & \text { otherwise }\end{cases} \\
\eta=\frac{\sum_{k \notin b e s t} \Delta_{i}^{(k)}}{\mid \text { best } \mid} \\
\text { best }=\left\{l / Q_{i}^{(l)} \geq Q_{i}^{(m)} \forall l, m \in[1, n]\right\}
\end{gathered}
$$

The above-mentioned $\Delta_{i}^{(j)}$ values are computed as shown in Equation 3. These $\Delta_{i}^{(j)}$ factors are computed from the relative difference between the quality of the best and the $j-t h$ techniques, $n$ being the number of available techniques. In this equation, $\xi$ represents a reduction factor, i.e., the ratio that is transferred from one technique to the other(s) (usually set to a value of 0.05 ). Finally, a minimum participation ratio can be established to guarantee that all the techniques are represented through all the search. This is done to avoid, if possible, premature convergence to undesired solutions caused by a technique that obtains all the participation in the early steps of the search and quickly converges to poor regions of the solution space, preventing the other techniques to collaborate at later stages of the process, in which they could be more beneficial.

$$
\Delta_{i}^{(j)}=\xi \cdot \frac{Q_{i}^{(b e s t)}-Q_{i}^{(j)}}{Q_{i}^{(b e s t)}} \cdot \Pi_{i-1}^{(j)} \quad \forall j \in[1, n] / j \neq b \epsilon s t
$$

To summarize, the presented algorithm works as follows. All the available techniques are allocated the same number of FEs at the beginning of the execution. At the end of each step, the quality of the new solutions created by each technique is evaluated and, based on this quality, its participation ratio is adjusted accordingly. This participation ratio is used to compute the number of FEs that each technique will be allowed to use in the next step of the search. If a minimum participation ratio has been established, then the number of FEs can not go below this threshold.

Finally, a restart mechanism, similar to the one used by the IPOP-CMA-ES algorithm, was also used within the proposed algorithm. With this strategy, the algorithm is halted whenever a restart stopping criteria is met, reinitializing the population and increasing its size by a factor of two until a maximum population size is reached. As this restart mechanism depends on some specific conditions of the IPOP-CMAES technique, the restart can only take place when this technique is being executed. However, the effect of the restart affects to all the available techniques, as it is the overall population which is restarted. Moreover, the framework easily allows the use of additional restart mechanisms associated to the remaining techniques or overall restart mechanisms independent of these techniques.

\section{EXPERIMENTAL PROCEDURE}

The results reported for this work have been obtained from 15 independent executions executed on the computer configuration displayed in Table 1.

Table 1: Computer Configuration

\begin{tabular}{|l|c|}
\hline PC & Intel Xeon 8 cores 1.86Ghz CPU \\
Operating System & Ubuntu Linux 8.04 \\
Prog. Language & C++ \\
Compiler & GNU C++4.3.2 \\
\hline
\end{tabular}

Regarding the parameter tuning, no thorough parameter study has been conducted for this work. The parameters of the algorithm were selected based on the extensive parameter tuning that was carried out for the $\mathrm{HRH}$ algorithm presented in [7] on a different testbed of functions, and for a similar study that considers the same benchmark of ISDA 2009 and experiments with a MOS based algorithm, submitted for publication to an international journal and currently ${ }^{1}$ under review. Table 2 displays the final values that were selected for this experimentation, both for the DE, the IPOPCMA-ES and also for the main algorithm. The parameters of the algorithms remain the same for all the functions and, thus, the Crafting Effort (CrE) value is zero.

Table 2: Parameters of the algorithm

\begin{tabular}{|l|c|}
\hline \multicolumn{1}{|c|}{ Parameter } & Value \\
\hline \hline Initial Population Size & 15 \\
Maximum Pop. Size (after restarts) & 6400 \\
DE CR & 0.5 \\
DE F & 0.5 \\
DE Crossover Operator & Exponential \\
DE Selection Operator & Tournament 2 \\
DE Model & classic \\
Minimum Participation Ratio & $5 \%$ \\
Number of Steps & 85 \\
\hline
\end{tabular}

\section{CPU TIMING EXPERIMENT}

For the timing experiment the proposed algorithm was run on $f_{8}$ for at least 30 seconds. This experimentation has been conducted on the aforementioned computer configuration depicted in Table 1 . The results of this study are reported in Table 3.

\begin{tabular}{ccccccc}
\multicolumn{8}{c}{ Table 3: } & CPU Timing \\
\hline D & 2 & 3 & 5 & 10 & 20 & 40 \\
runs & 182 & 161 & 143 & 112 & 82 & 52 \\
seconds $\times 10^{-6}$ & 3.5 & 3.9 & 4.5 & 4.5 & 4.7 & 5.8 \\
\hline
\end{tabular}

The CPU-time per function evaluation grows linearly up to 5 dimensions, probably due to the overhead of the hybridization procedures, and then it gets stabilized for dimensions 5, 10 and 20. Finally, for 40 dimensions, the CPU-time starts to grow again, this time due to the increased complexity for this problem size.

${ }^{1}$ March 2010 
Table 5: ERT loss ratio (see Figure 3) compared to the respective best result from BBOB-2009 for budgets given in the first column. The last row $\mathrm{RL}_{\mathrm{US}} / \mathrm{D}$ gives the number of function evaluations in unsuccessful runs divided by dimension. Shown are the smallest, $10 \%$-ile, $25 \%$-ile, $50 \%$-ile, $75 \%$-ile and $90 \%$ ile value (smaller values are better).

\begin{tabular}{|c|c|c|c|c|c|c|}
\hline & \multicolumn{6}{|c|}{$\boldsymbol{f}_{1}-\boldsymbol{f}_{24}$ in $\mathbf{5}-\mathrm{D}, \operatorname{maxFE} / \mathrm{D}=727186$} \\
\hline \#FEs/D & best & $10 \%$ & $25 \%$ & med & $75 \%$ & $90 \%$ \\
\hline 2 & 1.3 & 1.6 & 1.9 & 2.9 & 4.2 & 8.5 \\
\hline 10 & 2.3 & 3.3 & 3.8 & 5.1 & 6.8 & 50 \\
\hline 100 & 2.8 & 6.6 & 8.7 & 12 & 16 & 42 \\
\hline $1 \mathrm{e} 3$ & 2.2 & 2.2 & 3.2 & 9.3 & 25 & 51 \\
\hline $1 \mathrm{e} 4$ & 0.72 & 1.6 & 3.1 & 6.6 & 34 & $1.3 \mathrm{e} 2$ \\
\hline $1 \mathrm{e} 5$ & 1.2 & 1.6 & 3.1 & 5.6 & 17 & $2.5 \mathrm{e} 2$ \\
\hline $1 e 6$ & 0.52 & 1.2 & 2.6 & 4.7 & 16 & $2.7 \mathrm{e} 2$ \\
\hline RLus/D & $1 \mathrm{e} 5$ & $1 \mathrm{e} 5$ & $1 \mathrm{e} 5$ & $1 \mathrm{e} 5$ & $2 \mathrm{e} 5$ & $3 \mathrm{e} 5$ \\
\hline & \multicolumn{6}{|c|}{ 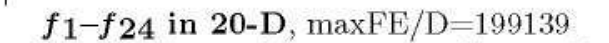 } \\
\hline$\# \mathrm{FEs} / \mathrm{D}$ & best & $10 \%$ & $25 \%$ & med & $75 \%$ & $90 \%$ \\
\hline 2 & 1.0 & 2.4 & 9.4 & 31 & 40 & 40 \\
\hline 10 & 4.8 & 6.9 & 10 & 51 & $2.0 \mathrm{e} 2$ & $2.0 \mathrm{e} 2$ \\
\hline 100 & 5.6 & 6.5 & 8.8 & 17 & 24 & 47 \\
\hline $1 \mathrm{e} 3$ & 0.26 & 1.6 & 3.2 & 9.8 & 44 & $1.4 \mathrm{e} 2$ \\
\hline $1 \mathrm{e} 4$ & 0.09 & 1.1 & 2.0 & 3.9 & 67 & $2.8 \mathrm{e} 2$ \\
\hline $1 \mathrm{e} 5$ & 0.07 & 0,94 & 2.1 & 4.0 & 14 & $2.2 \mathrm{e} 2$ \\
\hline $1 \mathrm{e} 6$ & 0.07 & 0.94 & 2.1 & 4.4 & 30 & $1.3 \mathrm{e} 2$ \\
\hline $\mathrm{RL}_{\text {US }} / \mathrm{D}$ & $1 \mathrm{e} 5$ & $1 \mathrm{e} 5$ & $1 \mathrm{e} 5$ & $1 \mathrm{e} 5$ & $2 \mathrm{e} 5$ & $2 \mathrm{e} 5$ \\
\hline
\end{tabular}

\section{RESULTS}

Results from experiments according to [4] on the benchmark functions given in $[2,5]$ are presented in Figures 1, 2 and 3 and in Tables 4 and 5 .

The overall results in the noiseless testbed are quite satisfactory in terms of achieved precision and scalability. The hybrid algorithm here presented is able to solve $24,24,24$, 24,21 and 20 functions out of 24 in $2,3,5,10,20$ and 40 dimensions, respectively.

Compared to the individual use of its composing algorithms, the hybrid algorithm obtains more stable results than any of them. Furthermore, functions $f 3$ and $f 4$ which are practically unsolvable for the IPOP-CMA-ES algorithm, are now solved thanks to the hybridization with the DE algorithm. On the other hand, the most difficult function for our approach is $f_{24}$, for which convergence is never reached for dimension 20 or above. Nevertheless, this is somehow reasonable, as this function has been designed to be deceptive for Evolution Strategies (and the DE is also unable to deal with it).

Furthermore, it can also be observed that the proposed algorithm achieves one of the best results in terms of ECDFs values, compared with the algorithms presented in the previous BBOB-2009 workshop, for all the groups of functions, as it can be seen in Figure 2.

Finally, regarding the number of Fitness Evaluations required to reach a particular precision, it can be higher than for other algorithms, such as the IPOP-CMA-ES when it is used individually. This is normal, as the regulatory mechanisms implemented by the MOS framework need some time to take a decission and adjust the participation of each technique accordingly.
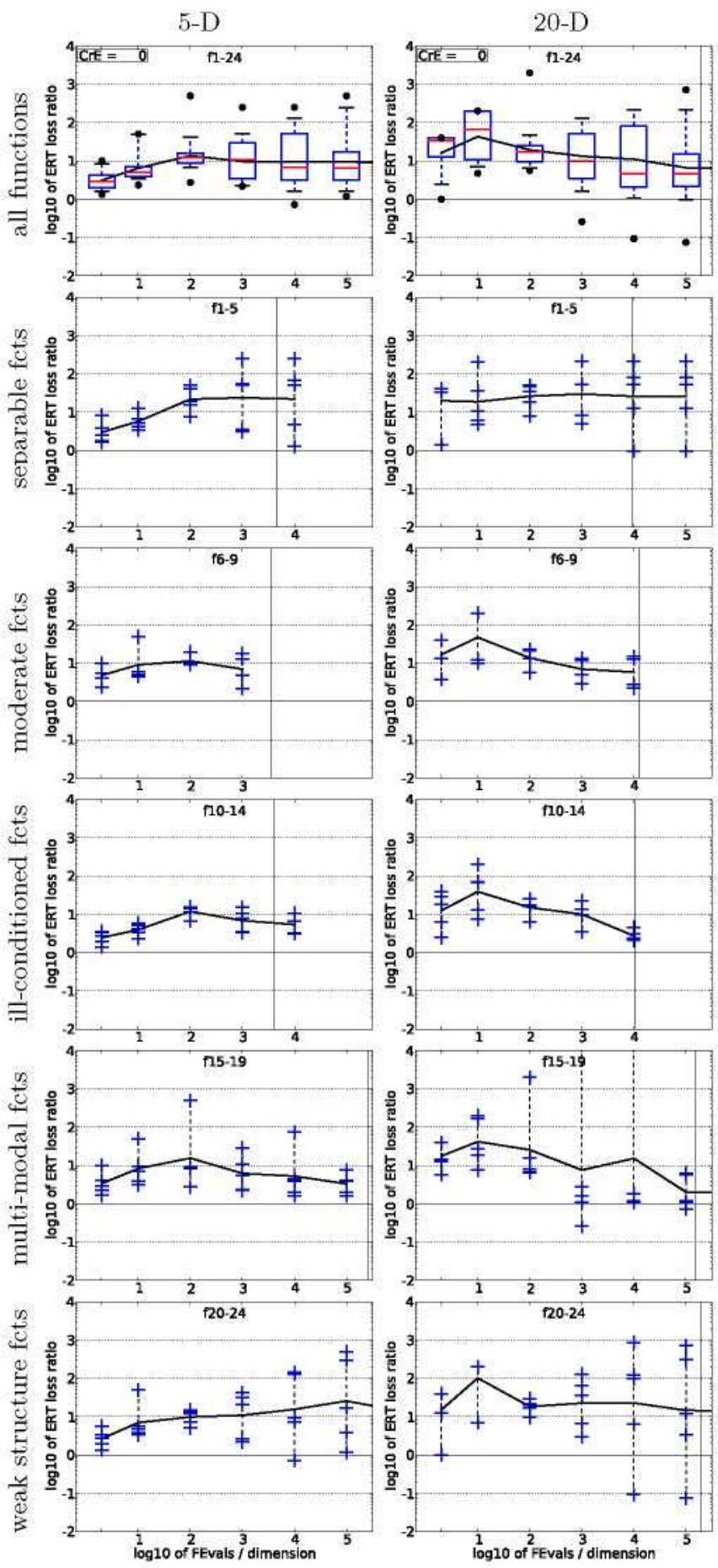

Figure 3: ERT loss ratio versus given budget FEvals. The target value $f_{\mathrm{t}}$ for ERT (see Figure 1) is the smallest (best) recorded function value such that $\operatorname{ERT}\left(f_{\mathrm{t}}\right) \leq$ FEvals for the presented algorithm. Shown is FEvals divided by the respective best ERT $\left(f_{\mathrm{t}}\right)$ from BBOB-2009 for functions $f_{1}-f_{24}$ in 5-D and 20-D. Each ERT is multiplied by $\exp (\mathrm{CrE})$ correcting for the parameter crafting effort. Line: geometric mean. Box-Whisker error bar: 25-75\%-ile with median (box), 10-90\%-ile (caps), and minimum and maximum ERT loss ratio (points). The vertical line gives the maximal number of function evaluations in this function subset. 

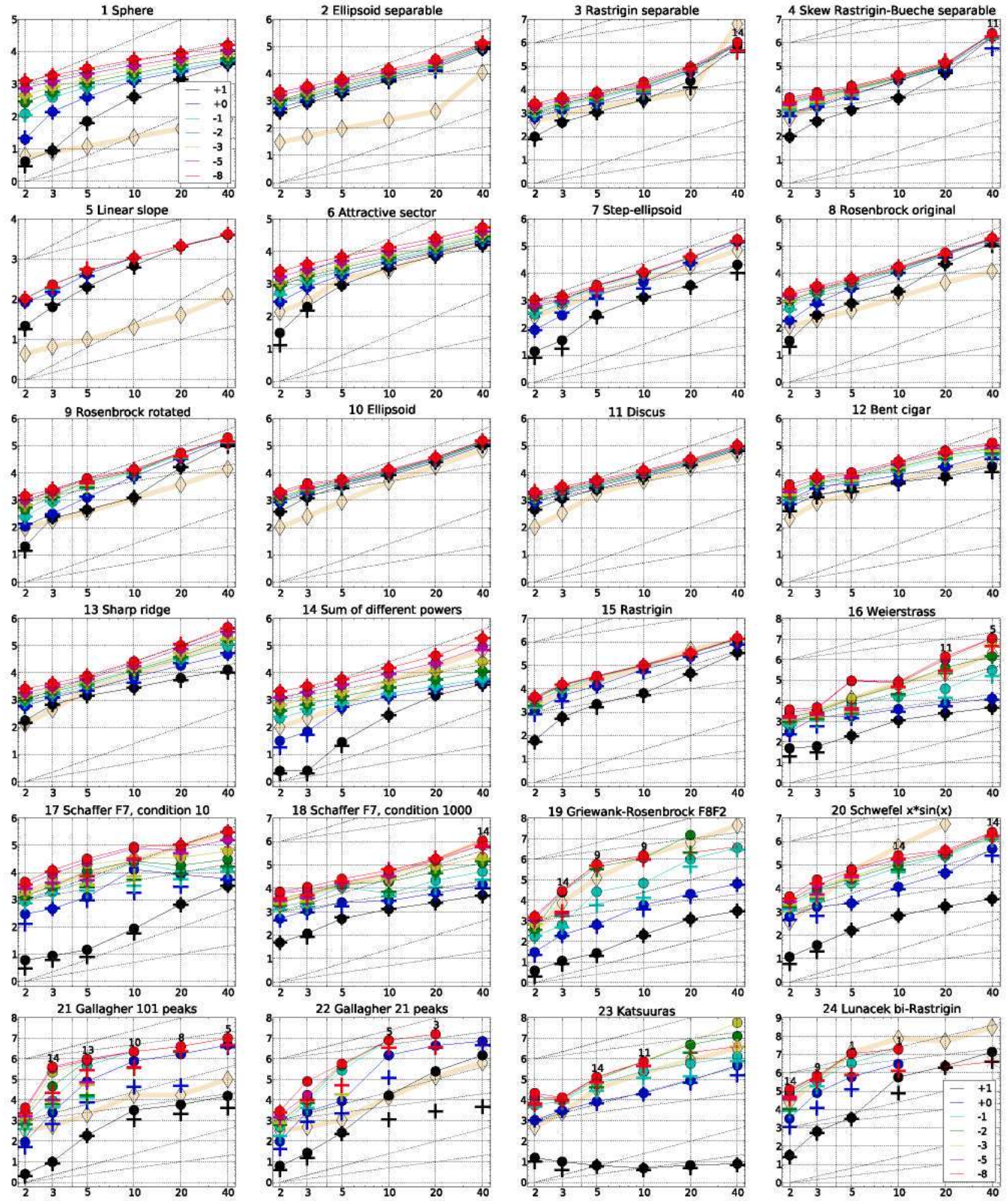

Figure 1: Expected Running Time (ERT, $\bullet$ ) to reach $f_{\text {opt }}+\Delta f$ and median number of $f$-evaluations from successful trials (+), for $\Delta f=10^{\{+1,0,-1,-2,-3,-5,-8\}}$ (the exponent is given in the legend of $f_{1}$ and $f_{24}$ ) versus dimension in log-log presentation. For each function and dimension, $\operatorname{ERT}(\Delta f)$ equals to \# $\operatorname{FEs}(\Delta f)$ divided by the number of successful trials, where a trial is successful if $f_{\mathrm{opt}}+\Delta f$ was surpassed. The \#FEs $(\Delta f)$ are the total number (sum) of $f$-evaluations while $f_{\mathrm{opt}}+\Delta f$ was not surpassed in the trial, from all (successful and unsuccessful) trials, and $f_{\text {opt }}$ is the optimal function value. Crosses $(x)$ indicate the total number of $f$-evaluations, \#FEs $(-\infty)$, divided by the number of trials. Numbers above ERT-symbols indicate the number of successful trials. Y-axis annotations are decimal logarithms. The thick light line with diamonds shows the single best results from $\mathrm{BBOB}-2009$ for $\Delta f=10^{-8}$. Additional grid lines show linear and quadratic scaling. 

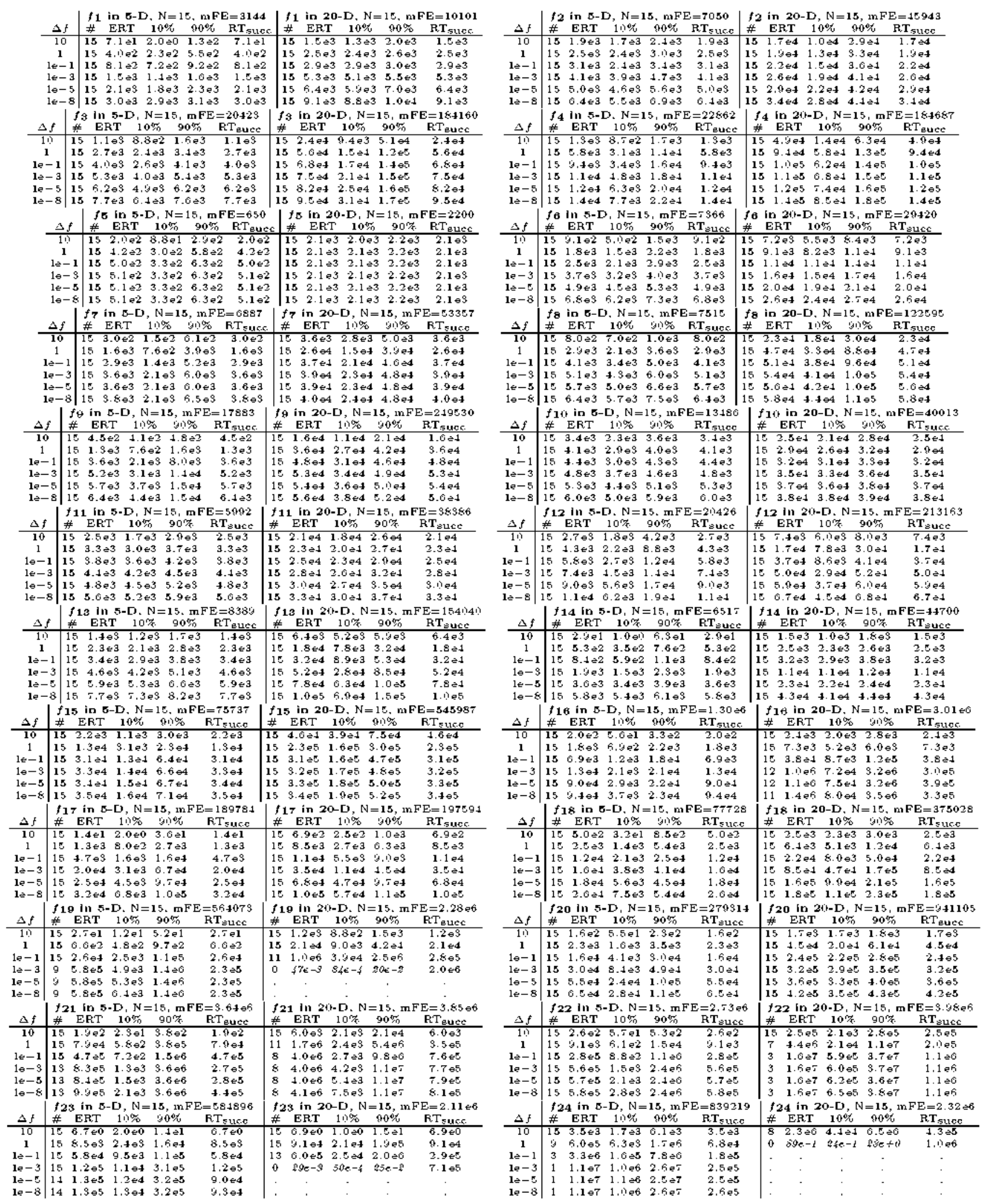

Table 4: Shown are, for a given target difference to the optimal function value $\Delta f:$ the number of successful trials (\#); the expected running time to surpass $f_{\circ \mathrm{pt}}+\Delta f$ (ERT, see Figure 1); the $10 \%$-tile and $90 \%$-tile of the bootstrap distribution of ERT; the average number of function evaluations in successful trials or, if none was successful, as last entry the median number of function evaluations to reach the best function value $\left(\mathbf{R T}_{\text {succ }}\right)$. If $f_{\text {opt }}+\Delta f$ was never reached, figures in italics denote the best achieved $\Delta f$-value of the median trial and the $10 \%$ and $90 \%$-tile trial. Furthermore, $\mathrm{N}$ denotes the number of trials, and mFE denotes the maximum of number of function evaluations executed in one trial. See Figure 1 for the names of functions. 

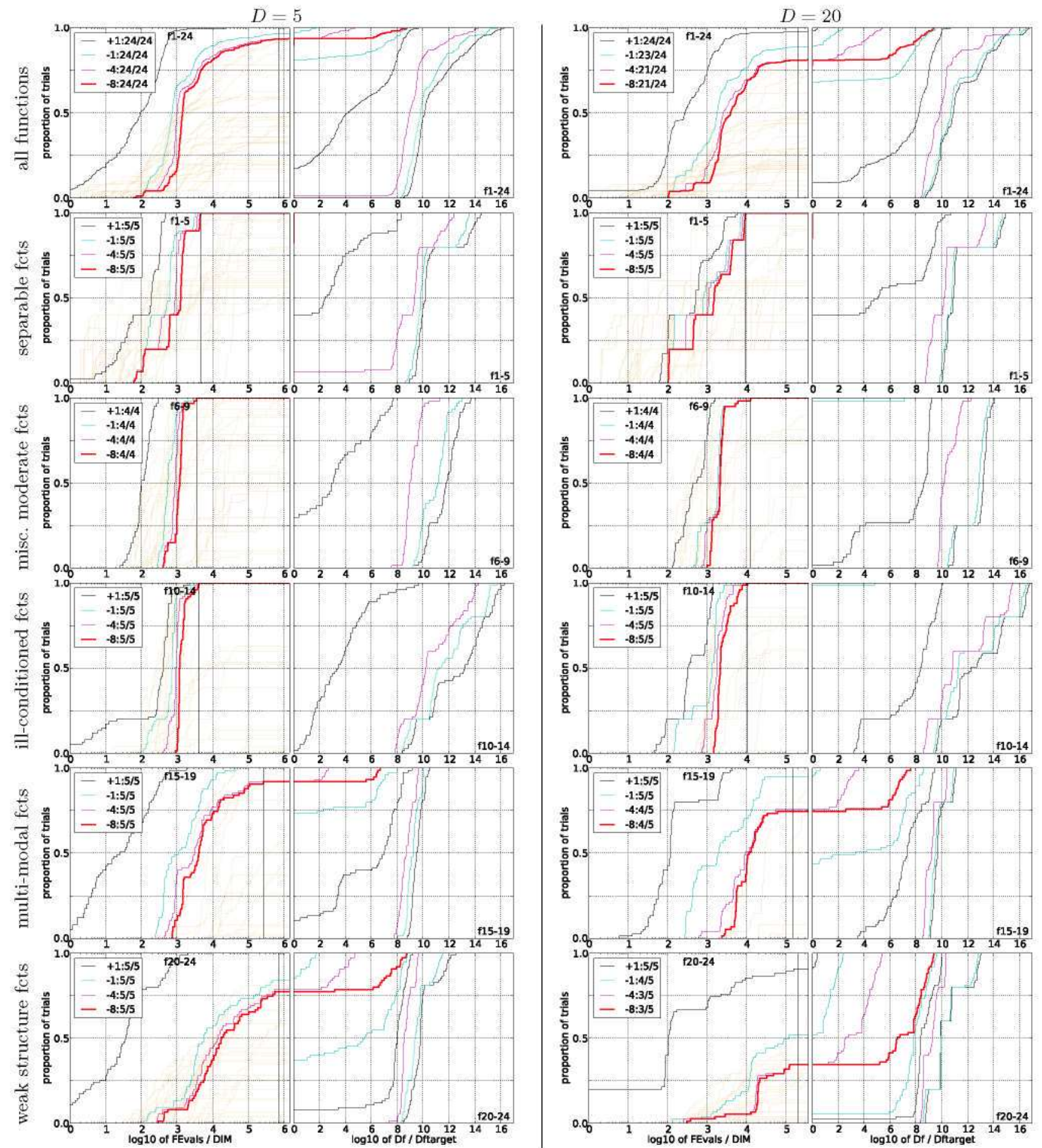

Figure 2: Empirical cumulative distribution functions (ECDFs), plotting the fraction of trials versus running time (left subplots) or versus $\Delta f$ (right subplots). The thick red line represents the best achieved results. Left subplots: ECDF of the running time (number of function evaluations), divided by search space dimension $D$, to fall below $f_{\text {opt }}+\Delta f$ with $\Delta f=10^{k}$, where $k$ is the first value in the legend. Right subplots: ECDF of the best achieved $\Delta f$ divided by $10^{k}$ (upper left lines in continuation of the left subplot), and best achieved $\Delta f$ divided by $10^{-8}$ for running times of $\mathrm{D}, 10 \mathrm{D}, 100 \mathrm{D} \ldots$ function evaluations (from right to left cycling blackcyan-magenta). The legends indicate the number of functions that were solved in at least one trial. FEvals denotes number of function evaluations, $D$ and DIM denote search space dimension, and $\Delta f$ and Df denote the difference to the optimal function value. Light brown lines in the background show ECDFs for target value $10^{-8}$ of all algorithms benchmarked during BBOB-2009. 


\section{CONCLUSIONS}

In this contribution, a hybrid algorithm combining Differential Evolution and IPOP-CMA-ES has been presented and benchmarked on the $\mathrm{BBOB}-2010$ noiseless testbed. The experimental results show a good performance on all the groups of functions and a good scalability. The proposed algorithm has been able to solve $24,24,24,24,21$ and 20 functions out of 24 in 2, 3, 5, 10, 20 and 40 dimensions, respectively. Furthermore, it obtains better results than its composing algorithms when used individually. Additionally, a comparative analysis with the algorithms presented at the BBOB-2009 workshop reveals that our approach obtains one of the best results in terms of convergence. Further research will investigate with new techniques to complement the two used algorithms in those functions in which the hybrid algorithms obtains worse results. A more thorough study on the control mechanisms, specially those related to the detection of the stagnation and the restart of the search process, could be also useful to increase the stability in those functions in which the convergence to the global optimum is not always obtained.

\section{ACKNOWLEDGMENTS}

This work was supported by the Madrid Regional Education Ministry and the European Social Fund and financed by the Spanish Ministry of Science TIN2007-67148. The authors thankfully acknowledge the computer resources, technical expertise and assistance provided by the Centro de Supercomputación y Visualización de Madrid (CeSViMa) and the Spanish Supercomputing Network.

\section{REFERENCES}

[1] A. Auger and N. Hansen. A restart CMA evolution strategy with increasing population size. In Proceedings of the IEEE Congress on Evolationary Computation, $C E C 2005$, pages 1769-1776. IEEE Press, 2005.
[2] S. Finck, N. Hansen, R. Ros, and A. Auger. Real-parameter Black-Box Optimization Benchmarking 2009: Presentation of the noiseless functions. Technical Report 2009/20, Research Center PPE, 2009. Updated February 2010.

[3] Y. Gao and Y.-J. Wang. A memetic differential evolutionary algorithm for high dimensional functions' optimization. In Proceedings of the Thind International Conference on Natural Computation (ICNC 2007), pages 188-192. IEEE Press, 2007.

[4] N. Hansen, A. Auger, S. Finck, and R. Ros. Real-parameter Black-Box Optimization Benchmarking 2010: Experimental setup. Technical Report RR-7215, INRIA, 2010.

[5] N. Hansen, S. Finck, R. Ros, and A. Auger. Real-parameter Black-Box Optimization Benchmarking 2009: Noiseless functions definitions. Technical Report RR-6829, INRIA, 2009. Updated February 2010.

[6] A. LaTorre. A Frameuork for Hybrid Dynamic Evolutionary Algorithms: Multiple Offspring Sampling (MOS). PhD thesis, Universidad Politécnica de Madrid, November 2009.

[7] S. Muelas, A. LaTorre, and J. Peña. A memetic differential evolution algorithm for continuous optimization. In Proceedings of the 9th International Conference on Intelligent Systems Design and Applications, ISDA 2009, pages 1080-1084. IEEE Press, November 2009.

[8] R. Stom and K. Price. Differential evolution - A simple and efficient adaptive scheme for global optimization over continuous spaces. Technical report, 1995.

[9] E.-G. Talbi. A taxonony of hybrid metaheuristics. Journal of Heuristics, 8(5):541-564, September 2002 . 